$Q P$

111

LA

Biology

U.C. BERKELEY LIBRARY

UC-NRLF

BIOLOGY LIBRARY USB

+5 $25>7 ?$ 





\section{INTERPRETATIONS OF THE INITIAL PHASES OF THE ELECTROCARDIOGRAM WITH SPECIAL REFERENCE TO THE THEORY OF "LIMITED POTEN- TIAL DIFFERENCES"}

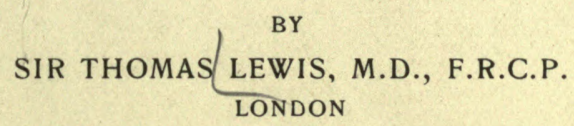

\footnotetext{
SEVENTH MELLON LECTURE, DELIVERED BEFORE THE SOCIETY FOR BIOLOGICAL RESEARCH, UNIVERSITY OF PITTSBURGH SCHOOL OF MEDICINE, MAY 8, 1922.
} 
$\because \because \quad \because 4$

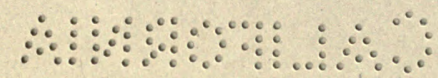




\title{
MELLON LECTURE
}

(UNDER THE AUSPICES OF THE SOCIETY FOR BIOLOGICAL RESEARCH)

\author{
UNIVERSITY OF PITTSBURGH \\ SCHOOL OF MEDICINE
}

SEVENTH LECTURE

INTERPRETATIONS OF THE INITIAL PHASES OF THE ELECTROCARDIOGRAM WITH SPECIAL REFERENCE TO THE THEORY OF "LIMITED POTENTIAL DIFFERENCES"

BY

SIR THOMAS LEWIS, M.D., F.R.C.P. 
and

QPIII

L4

$y^{N^{2}}$ 


\title{
INTERPRETATIONS OF THE INITIAL PHASES OF THE ELECTROCARDIOGRAM WITH SPECIAL REFER- ENCE TO THE THEORY OF "LIMITED POTENTIAL DIFFERENCES” *
}

\author{
SIR THOMAS LEWIS, M.D., F.R.C.P. \\ LONDON
}

The subject chosen for this lecture is one on which much has been written in recent years, but upon which there is still no complete consensus of opinion. In a review ${ }^{1}$ written about three years ago I attempted to explain the constitution of the electrocardiogram on a new basis and put forward views of a novel kind. These views have been accepted by a number of workers; from other workers they have received criticism, publicly or privately expressed. The lack of uniform conclusions, I think, results largely from misunderstandings, such misunderstandings as may be expected to arise when the subject discussed is not only intricate but concerned with the ultimate processes of cellular activity and its manifestations. In studying the electrocardiogram we attempt to explain its general deflections in rational terms, yet the terms which we are forced to employ are terms which refer to intimate and intangible effects of cellular physics and chemistry. Although, as may be acknowledged at once, I have experienced recurring difficulty in my attempts clearly to conceive the manner in which the electrical events are related to events in the muscle; yet the conception at which I arrived some few years ago and which is now reconsidered in the light of recent discussion and observation has not altered fundamentally, though it may, perhaps, have grown a little clearer during the interval. In attempting a more distinct statement of the case it seems desirable to state that while I refuse to stand committed, once and for all, to these views, yet our present knowledge does seem to necessitate movement from the older hypotheses in some such direction as will be indicated.

In discussing the question before us one rule stands out as paramount in importance; the facts and the hypotheses must be divided from each other as scrupulously as possible. Hypothesis cannot be avoided, per-

* Seventh Mellon lecture.

* Working on behalf of the British Medical Research Council at University College Hospital Medical School, London. Delivered before the Society for Biological Research, School of Medicine, University of Pittsburgh, May 8, 1922.

1. Lewis: The Mechanism and Graphic Registration of the Heart Beat, London, 1920. 
haps never will be avoided, in a full consideration of our problem, and it would be presumptuous if my views were brought before you as views approaching to a final solution. Nevertheless, by carefully weighing our present knowledge, certain final statements may be made, certain statements may be denied, and we can bring our conceptions a step

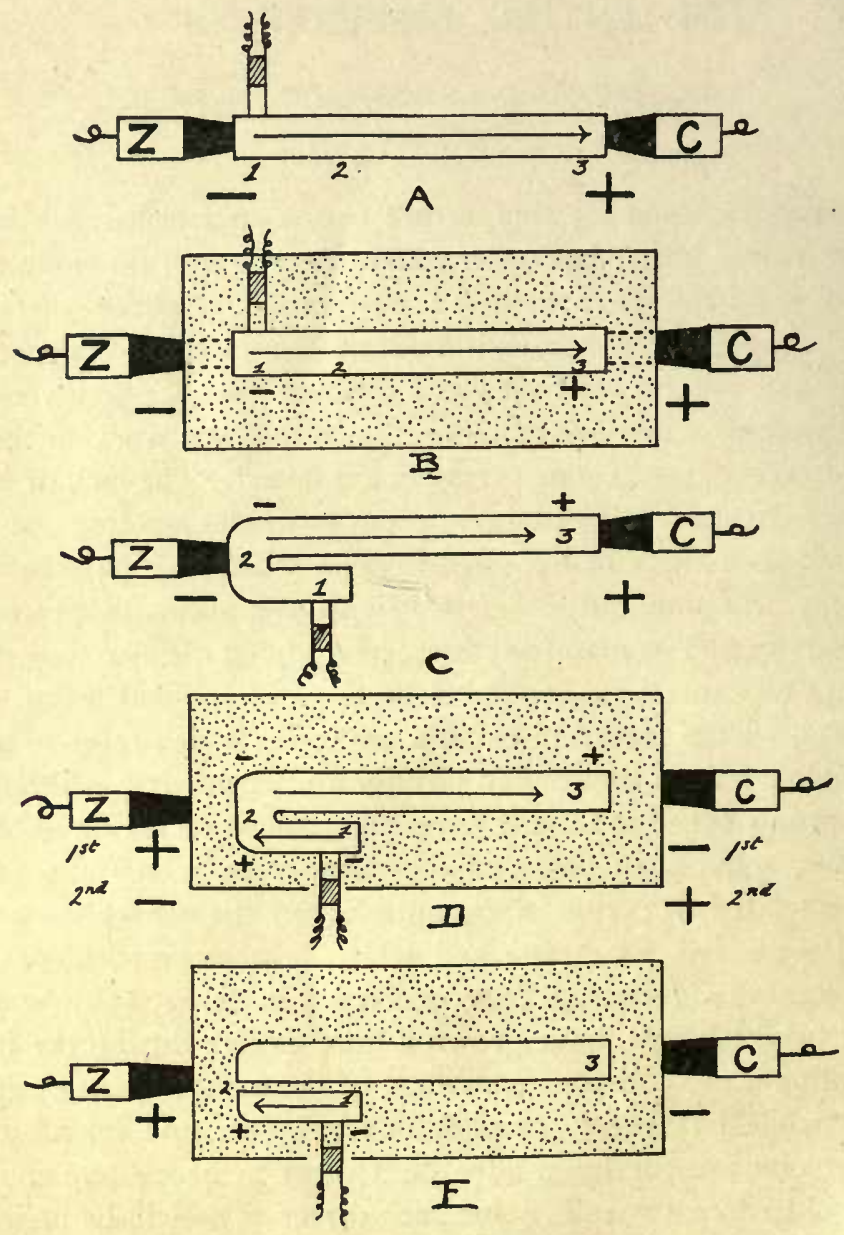

Fig. 1.-Diagrams, illustrating the potential differences in simple muscle strips, activated at one end.

nearer clarity and truth. Of one thing I feel sure, namely, that a close consideration of the views expressed, and further observations tending either to support or to destroy them, will prove fruitful.

In considering the meaning of the electrocardiogram, we have all started from a common basis. By common consent, if you place two leading-off electrodes directly on the two ends of a narrow strip of 
uninjured muscle (Fig. $1 \mathrm{~A}$ ) and now stimulate the muscle to contract in the region of one contact, the galvanometer shows the development of a difference in potential between the two contacts, that which lies at the point stimulated $(Z)$ becoming negative relative to its fellow $(C)$. The galvanometer shows us that an electromotive force is developed between these two points; current flows from one to the other through the muscle and through the galvanometer. This experiment, easily performed, has. been carried out by very numerous observers with uniform results; the same event is witnessed whether voluntary or cardiac muscle is used, but it is the last only which I shall ask you to hold in mind during this lecture, for it is on cardiac muscle alone that I feel competent to speak, and in respect of which alone I shall draw my conclusions. It is on this experiment that the conclusion, which we have all accepted, is based, namely, that cardiac muscle entering the active state shows relative negativity to muscle which is inactive. But although this observation is easy to confirm, and although the conclusion derived from it does not seem open to question, I believe that it often gives rise to misconception when the meaning of the electrocardiogram is considered in the light of it.

The way in which the chief misconceptions arise I will endeavor to explain ultimately. Meanwhile, it should be remarked that in the simple experiment described the electrodes are placed against very small areas of the muscle strip; this method of leading chiefly signals the events which occur at the ends of the muscle, rather than the events in its whole length. Further, it is to be noted that when a difference of potential arises between the two ends as a result of activity of one end, the current flow is necessarily confined to the line of the muscle; for the muscle is isolated. The line of the muscle represents the line of the electrical axis, or the line of maximal potential difference.

Let us now take a second example (Fig. $1 \mathrm{~B}$ ), one which, in the simple form illustrated, is theoretical, but one which would be more comparable to the experiment of electrocardiography. Imagine a strip of cardiac muscle completely embedded in a moist conducting substance, and that our contacts are placed, not immediately on the ends of the strip, but on the substance in which the muscle lies embedded. So they lie in human electrocardiography. If now the muscle end 1 becomes active, the galvanometer will again record current flow. The muscle end 1 we know becomes relatively negative to the muscle end 3 ; the contacts $Z$ and $C$ will no doubt reflect this change. If you wish to do so you may regard the contacts as prolonged through the medium in which the muscle is embedded, to the nearest points of the muscle, $i$. e., to the original ends 1 and 3 . There is this contact, but it is not the sole contact, for our electrodes are now connected, not only to the ends of the muscle, but to its whole surface. This difference 
between the two methods of leading off should not influence the direction of the first potential difference exhibited by contact $C$ and $Z$, when, as in our illustration, we are dealing with a straight and narrow strip of muscle lying in one line between the contacts; but as we shall see, it becomes of consequence when the relation of muscle to contacts is altered. A second difference between the two methods of leading off is also to be noted. When, in the last experiment, the muscle is embedded in a conducting substance, the current flows not only through the muscle and galvanometer; it flows through the surrounding medium. In other words, we have introduced a series of shunts along the borders of the muscle and these decrease the amount of current flowing through the galvanometer. While we may expect the movement of the galvanometric recorder to maintain its original direction (contact $Z$ showing relative negativity to contact $C$ ), we may not expect its movement to be so considerable.

I emphasize the fact that the comparison made is between an actual experiment (Fig. 1 A) and a theoretical experiment (Fig. $1 \mathrm{~B}$ ). The actual events in the second experiment (Fig. $1 \mathrm{~B}$ ) have, I think, not been recorded, though there is sufficient evidence based on observation of a less direct kind to convince us that the curves obtained in the first and second experiment will have the same general form in their initial phase. That there will be this smilarity is, I think, generally recognized; it is not a matter in dispute. But this conception is apt to lead up to a second, namely, that curves taken by the two methods are built up in identical fashions; and that the two methods of leading will in all circumstances yield similar results. They may do so when a simple and narrow strip of muscle is placed in line with the leading off contacts; but they do not, I believe, in all circumstances. Suppose that the unembedded muscle is bent on itself at one end and that the contacts are placed at 2 and 3 (Fig. 1 C). Again, there will be agreement as to the effect produced. Soon after 1 is stimulated, a current will flow through the galvanometer in the original direction, indicating that contact $Z$ has become relatively negative to $C$. This change is set up when the muscle on which contact $Z$ lies becomes active. It is not produced by activity of the muscle strip between 1 and 2 ; activity in this portion of the muscle has no material effect since the free end 1 is not connected to the leading off contact $C$. This illustration serves to emphasize the fact that when contacts are placed directly on the muscle, that it is an event occurring in the muscle immediately under the proximal contact which is responsible for the first swing of the recording instrument.

We come next to the fourth and crucial example, the muscle bent on itself and embedded (Fig. 1D). In these circumstances how will the current first flow? I have asked this question of many physiologists 
during the past six years, and have obtained almost always the reply that the direction of current flow will be the same as in the last example. It is said that 1 will become negative relative to 3 , and that consequently $Z$ will show negativity relative to $C$. Now, this reply is an assumption ultimately based on the belief that the two methods of leading off are sufficiently similar; actually the experiment has not been performed in this simple form. For a long while I have felt unable to accept this view of what will happen. The view is inconsistent with certain experiments which will be described presently. The view which I put forward is that relative negativity will first develop at contact $C$; and it will develop at $C$ because, when 1 becomes active, we are leading off from the embedded strip of muscle 1 to 2 , and the remainder of the strip 2 to 3 does not for the moment concern us. It does concern us of course when the excitation wave in traveling along the strip moves into the region between 2 and 3 ; when the wave in traveling has turned the corner, $Z$ will become relative to $C$, the flow of current becoming in consequence reversed. But for the moment we shall do well to concentrate attention on the first phase of the reaction, for this is in dispute.

The statement just made in respect of the first phase, brings us I think to the critical arguments of the discussion, namely, does the prolongation of the strip from 2 to 3 effect the reaction shown by the galvanometer when 1 becomes active. The usual view has been that it will. My own view is that it does not.

You may ask, why the actual experiment is not performed? The reply is that it cannot be carried out in so simple a fashion on cardiac muscle which is uninjured, and that if you use injured muscle, your experiment is open, on theoretic grounds, to serious criticism. But, as I hope later to show, the experiment can be performed under somewhat more complex conditions, and that it yields the results which I anticipate.

The argument turns as stated on the influence or noninfluence of the portion of muscle 2 to 3 , on the initial electrical reaction. According to one view, that which seems so far to have obtained the greater number of direct or indirect supporters, you are to consider 1 as relatively negative, and to consider the whole of the remainder of the muscle as relatively positive. This view implies that in determining the direction of flow, you are to join the center of the active mass of muscle to the center of the inactive mass. According to the other view, you are to consider the part of the muscle which is becoming active as relatively negative, and the inactive muscle in union with it and in its immediate vicinity as relatively positive. I put forward this second view because it is consistent with and explains my experiments and because so far as I am aware it is inconsistent with no other experimental facts; the first view, though it is admittedly consistent with many experiments, is 
not consistent with all. For the sake of brevity we may term the first hypothesis that of "distributed potential differences," the second that of "limited potential differences."

At this point it may, perhaps, be appropriate to allude to my previous review. You will perceive that, if the hypothesis of limited potential differences is correct, the direction in which the current sets in the muscle will be the direction in which the excitation wave is at that moment traveling, for it will always move from the point which is becoming active to inactive muscle in the immediate vicinity of the latter. This association, which I believe to be an absolutely constant one, ${ }^{2}$ is one on which I have laid stress; in doing so I have tended to imply that the first is dependent on the second. Such has not been my meaning though it has been inferred from what has been, perhaps, an unfortunate phraseology, and this inference is perhaps chiefly responsible for misconception of my hypothesis. To state that the direction which the excitation wave takes in traveling governs the form of the corresponding curve, ${ }^{3}$ cannot be considered fundamentally sound.

A more correct expression would be that both are governed by one series of events in the muscle, and in consequence are definitely associated. It has been a matter of descriptive convenience to associate these two phenomena, direction of movement and direction of current flow. But because the meaning may be inferred that one is responsible for the other, I shall endeavor to avoid this method of description in my subsequent remarks.

Returning to the question at issue, namely, the influence of outlying muscle on the electrical reaction, let us consider the case of two entirely separate masses of muscle (Fig. $1 \mathrm{E}$ ). Under this arrangement, if 1 becomes active, the contacts at $C$ will become negative to the contact $Z$. Here no one argues that the presence of the inactive muscle strip 2 to 3 will influence the result, it becomes part of the moist substance in which the strip 1 and 2 is embedded. To illustrate this statement, take the example of complete heart block. When the auriculoventricular bundle is divided experimentally or by disease, the electrocardiogram records the independent activities of auricle and ventricle. Each chamber gives its separate curve, but the curves of one and the other fall in

2. I would here express the view that, unless the conditions are very simple, the direction in which the current is setting in a muscle strip as a whole is not to be ascertained with certainty from an examination of two small points of contact on its surface (as in Fig. 1A); but in my view it can be ascertained by the method of leading which Fig. 1B illustrates. When I say that the association between the set of current and direction of travel is constant, I wish to refer to direction of current as ascertained by the method of indirect leading (method of Fig. 1B) and by this method only.

3. The phrase actually used in "The Mechanism and Graphic Registration of the Heart Beat," London, 1920. 
varying relationship to each other. It is quite clear from such records that activity or inactivity, partial or complete, in one chamber has no influence on the shape or amplitudes of the electrical currents derived from the other (Fig. 2). Providing that we are dealing with separate masses of heart muscle, the electromotive forces set up in one chamber are not influenced by those set up in the other. The forces developed in the two chambers find full and individual expression; in explaining such curves we do not balance activity in one chamber against inactivity in the other.

In regard, therefore, to the illustration (Fig. 1E) we are safe in assuming that the activation of strip 1 to 2 at point 1 will yield an electromotive force having a certain direction and amplitude, independently of what is happening in the strip 2 to 3 . How does this arrangement of the muscle fibers differ from that illustrated by Figure 1D.

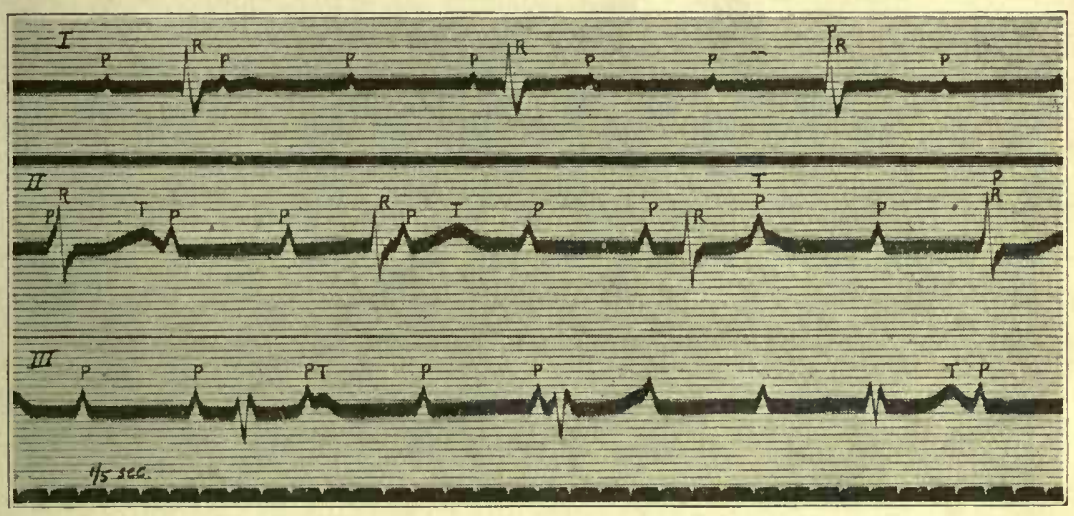

Fig. 2. Electrocardiograms from a case of complete heart block, and taken by means of Leads I, II and III.

In one respect only; in the first illustration there is functional union between the two strips at the point 2 . If 1 is stimulated to activity in these two sets of circumstances, will the initial resultant swings of the galvanometer be in opposite directions; will they indicate relative negativity at $Z$ in one case and at $C$ in the other? According to the hypothesis of distributed potentials this will happen. But in both instances, when 1 becomes active, the strip 2 to 3 is recognized to be in a state of inactivity. The distribution of potentials is the same in both, unless we are to assume that when the two strips are part of one undivided piece of muscle, an active change happens in strip 2 to 3 , rendering it relatively more positive to 1 . In other words, if we are to accept the hypothesis of distributed potentials, it is essential to assume that, from its initiation, active change produced by stimulation does not confine itself to that end of the muscle which is stimulated. I know of 

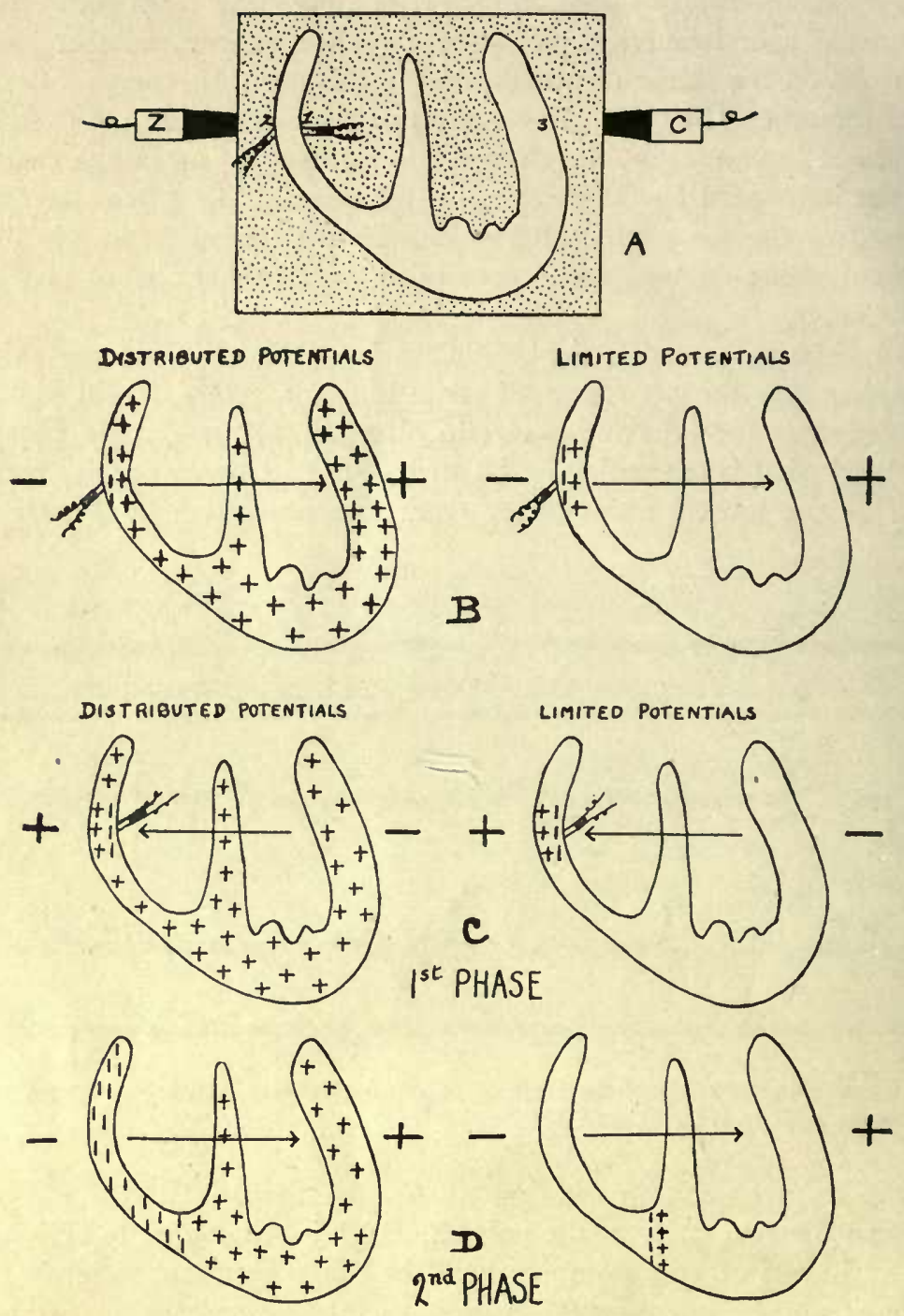

Fig. $3 \mathrm{~A}$ and $\mathrm{B}$.-When the heart is stimulated at its epicardial surface (Fig. $3 \mathrm{~B}$ ), contacts placed in line with the point stimulated show first of all an electrical relation which is indicated by the large + and - signs. This reaction of the contacts is explained alternatively by the hypotheses of distributed and limited potential differences (see small + and - signs of the diagram). In this example the distribution of potentials in the muscle and under the contacts is consistent, irrespective of the hypothesis chosen. A, C and D: The heart is stimulated at its endocardial surface and the potential difference recorded by the contacts is at first in one direction (phase 1) and later becomes reversed (phase 2). The supposed potential differences in the muscle, during the second phase, are consistent with what is found at the contact points, irrespective of the hypothesis adopted; but this is not so for the first phase; in this the hypothesis of limited potential differences is alone consistent with observation. 
no evidence in favor of such a diffused effect; on the contrary, the argument based on the fact that in one instance we are dealing with a single piece of muscle and in the other case with separate pieces of muscle, though plausible, can be shown to be unsound. The experiment of the bent piece of muscle can be performed on uninjured heart muscle. It is accomplished as follows : Leading off contacts are placed on a dog's chest wall (Fig. 3A), the one on the right side, the other on the left, and the right ventricle is stimulated first on its epicardial surface at 2 , and secondly on its endocardial surface at 1 , the points stimulated being in the same line as the leading off contacts. It will be obvious that, when we stimulate at 2 , the excitation wave will move in a general direction in the body from right to left across the heart (from 2 toward 3 ). It will be equally obvious that if we stimulate at 1 the excitation will at first move in a general direction from 1 toward 2, and that a little later the general course from 2 toward 3 will be pursued. In the

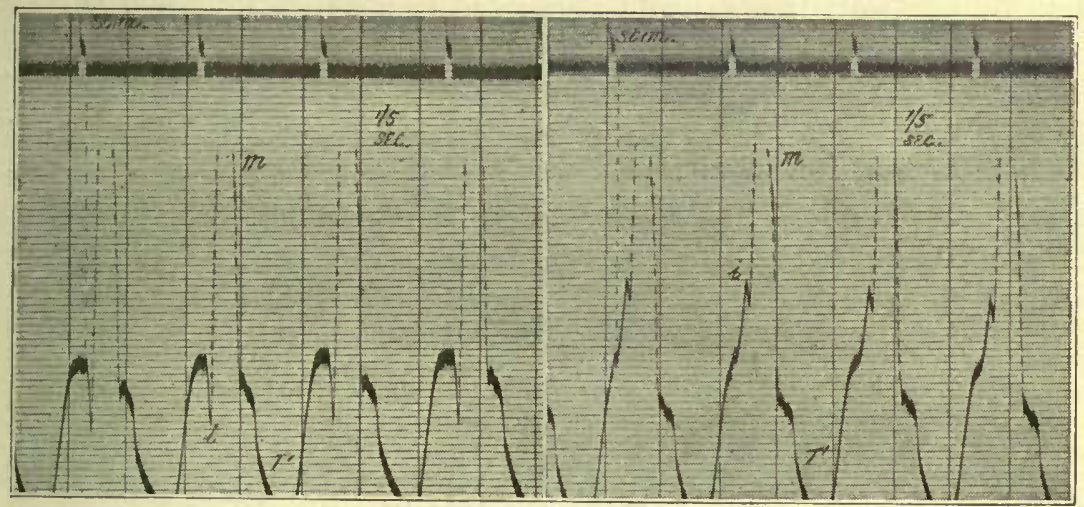

Fig. 4. An example of the findings of four distinct experiments. The lead was from the right chest wall ( $Z$ contact) to the left chest wall ( $C$ contact) and the wall of the right ventricle was stimulated on its epicardial surface (right hand record), and on its endocardial surface (left hand record). In the last case a small pair of long insulated electrodes was introduced through a small slit in the wall of the conus or through the jugular vein. The two points of stimulation (inside and outside) lay no more than $0.5 \mathrm{~cm}$. from each other. The curves differ in one respect, the left hand curve begins with an initial phase $i$ directed downward, in the right hand curve this phase is directed upward. These initial phases of the curves correspond to involvement of the wall at the point stimulated (the involvement being in opposite directions in the two circumstances). The main deflection $m$ is similar in both curves; it represents spread from right to left in the ventricles as a whole. Curves of this kind are obtained most successfully if the lungs are well inflated so that good contact is maintained between the sides of the heart and the chest wall. The curves represent responses to rhythmic break shocks (single shocks give similar effects). The stimulus is recorded electrically in the top line. The curves are not distorted by the stimuli, as the time relations of stimulus and shock show; reversal of the direction in which the stimulus entered the heart did not affect the form of the curves. 
first case the general direction in the body will be from right to left throughout the whole excitation of the ventricle; in the seconsl case, it will be for a brief period from left to right, and eventually from right to left. The experiment affords the general condition illustrated by the straight and bent muscle strips of Figure $1 \mathrm{~B}$ and $\mathrm{D}$. The result of this experiment is instructive. Stimulate the epicardial surface and, from the first, contact $Z$ is negative to contact $C$. This finding is consistent with both hypotheses, the potentials being distributed as shown in Figure $3 \mathrm{~B}$ and in Figure $4 .^{4}$ Stimulate the endocardial surface (Fig. $3 \mathrm{C}$ and $\mathrm{D}$ ) and the curve has two opposite phases, a short first phase during which contact $C$ is negative to contact $Z$, and a longer second phase during which contact $Z$ is negative to contact $C$ (Fig. 4). This finding is consistent with one of our hypothesis only, namely, that of limited potential differences. The association between the set of the current and the direction in which the excitation wave moves will be noted in this illustration. It constitutes a single and new illustration of an association which I believe to be firmly established and of which numerous examples have already been published. ${ }^{5}$ It was because I found this association constantly to exist in the normal heart beat of amphibians, reptiles, birds and mammalia, classes in which the spread of the excitation wave is very diverse, that I first recognized the hypothesis of distributed potentials to be untenable, for it is inconsistent with this association, as the diagrams clearly show (Figs. 3 $\mathrm{C}$ and D).

It may suffice at the present time if a few of the simpler illustrations are cited. The first of these permits the display of some important fallacies. It is the instance of the normal amplibian heart beat. The amphibian heart (Fig. 5) comprises not only sinus, auricle and ventricle, but also a bulbus arteriosus. It is on hearts of this or of a closely allied type, that almost the whole of the preliminary explanations of the electrocardiogram have been based; and it is with the mistaken interpretation of these curves, and the hypotheses derived from them, that we have today chiefly to contend. In this early work the heart was examined by placing on the base and apex of the ventricle two contacts and connecting them to a galvanometer. Because in such curves as were regarded to be typical, the first deflection indicated relative negativity of the base, it was concluded that the base first becomes active; it was further concluded that the excitation wave spreads as a simple wave from base to apex. Now, further and closer observation by modern

4. In stimulating the epicardial surface the curve is not usually expressed as a single phase. It rises a little, hangs or falls away as the endocardial lining is approached, and then rises steeply to yield the chief deflection which represents rapid involvement of a large mass of the ventricular substance.

5. Phil. Tr. Roy. Soc., B. 207:221, 1916. 
methods has shown that the first of these conclusions is usually incorrect, and that the last conclusion is never true. These early observations on the cold-blooded heart and the conclusions derived from them are open to serious criticism. The direction of the first deflection does not, in point of fact, always indicate primary negativity of the base. The direction depends largely in a given animal on the point chosen at the base for examination; there is also much variation from animal to antmal. Later observations have shown that sometimes a basal point is active before the apex; sometimes it becomes active after the apex (Fig. 5) ; sometimes certain points at the base are activated before, and certain points after, the apex. In all cases the base and apex are activated within a very short time interval of each other. The excitation first reaches the surface of the heart at neither base nor apex, it reaches

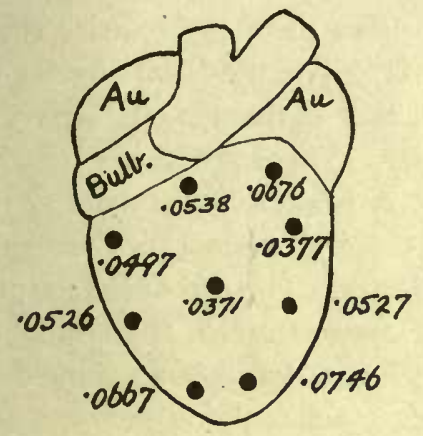

$A$

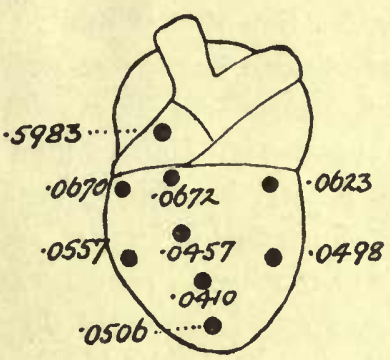

$E$

Fig. 5. - Outline diagrams of the hearts of Bufo vulgaris major, showing a number of surface readings expressed (in decimal points of a second) to the beginning of $R$ in an axial lead. Toad $\mathrm{A}$, an unpublished figure, showing that the base is activated earlier than the apex. Toad $\mathrm{E}$ (after the original figure) showing that the apex is activated earlier than the base. In both cases the central region of the ventral surface is activated earliest of all. In the axial electrocardiogram of each animal the chief deflection was an upright $R$; in the case of toad $\mathrm{E}$, the electrocardiogram also showed a prominent $S$ wave.

the central portions of the ventricle (Figs. 5 and $6 \mathrm{~A}$ ) and races simultaneously up to the base and down to the apex; the race may be won in one other direction; usually it is won at the apex. It is easy to understand why these base-apex curves of early days often seemed to indicate primary negativity of the base. The extreme base does become active a little before the apex in some amphibian hearts, though that is not the rule. If the extreme apex is chosen for one contact, and a point somewhat removed from the base is chosen for the second, and this would be the natural tendency ${ }^{\circ}$ of experiment, primary negativity

6. The tendency being to allow an interval of lesser or greater extent between the contact and the $A-V$ junction. 
of the base would usually be manifested; for the reason that a point a little removed from the base is usually activated earlier than a point at the extreme apex. The precise time relations of basal and apical activity being unknown beforehand, this observation would naturally be construed as indicating that the excitation wave starts at the base. From this conclusion, false as it was, it was an easy step to the still very prevalent general erroneous assumption that when, as is the rule, the basal contact of an indirect lead (method of Fig. 1 B) first demonstrates negativity, activity is confined to muscle which lies nearer to this basal contact than to the apical one. Thus, in human electrocardiography it has frequently been assumed that $R$, the chief deflection, is essentially a basal effect and that $S$ is essentially an apical effect; for $R$ represents relative negativity of the basal and $S$ of the apical contact. Neither conclusion is justified.

These assumptions, erroneous as I believe them to be, arise chiefly from the idea that if muscle at the base is active, the basal contact will show relative negativity and, conversely, that if the apex is active, the apical contact will show relative negativity. These are views based on the hypothesis of distributed potentials. A primary basal activity may display itself in relative negativity of the apical contact as the experiment illustrated in Figure $3 \mathrm{~A}$ clearly indicates. The erroneous assumptions also arise, in part, because the base-apex curve of the amphibian, where contacts are laid directly on the heart, has been assumed too rigidly to be comparable with the curves from outlying contacts such as are used in clinical electrocardiography. The difference between the two methods is that in the first the curve chiefly expresses the potential differences between two small areas, one at the base and one at the apex; while in the clinical method the lead (when axial) is from the whole of the basal and the whole of the apical parts of the heart; the clinical curves express the electrical changes in the entire mass of cardiac muscle; the direct leads do not. The two methods are strictly speaking incomparable (compare Figs. 1C and D from this point of view).

The axial electrocardiogram of the amphibian heart (Fig. 7), in so far as it expresses spread of the excitation wave, consists of the following deflections.

1. A chief and primary deflection $(R)$ indicating relative negativity of the contact beyond the base.

2. An inconstant second deflection $(S)$ indicating relative negativity of the contact beyond the apex.

3. A deflection $(B)$, occurring at a late phase of the electrocardiogram, corresponding to activation of the bulbus arteriosus, and indicating relative negativity of the apical contact. 
Let us deal briefly with the last deflection first. This bulbus deflection is due to the activity of muscle lying at the base of the ventricle; yet its direction indicates relative negativity of the apical contact. This observation clearly warns us against lightly concluding that relative
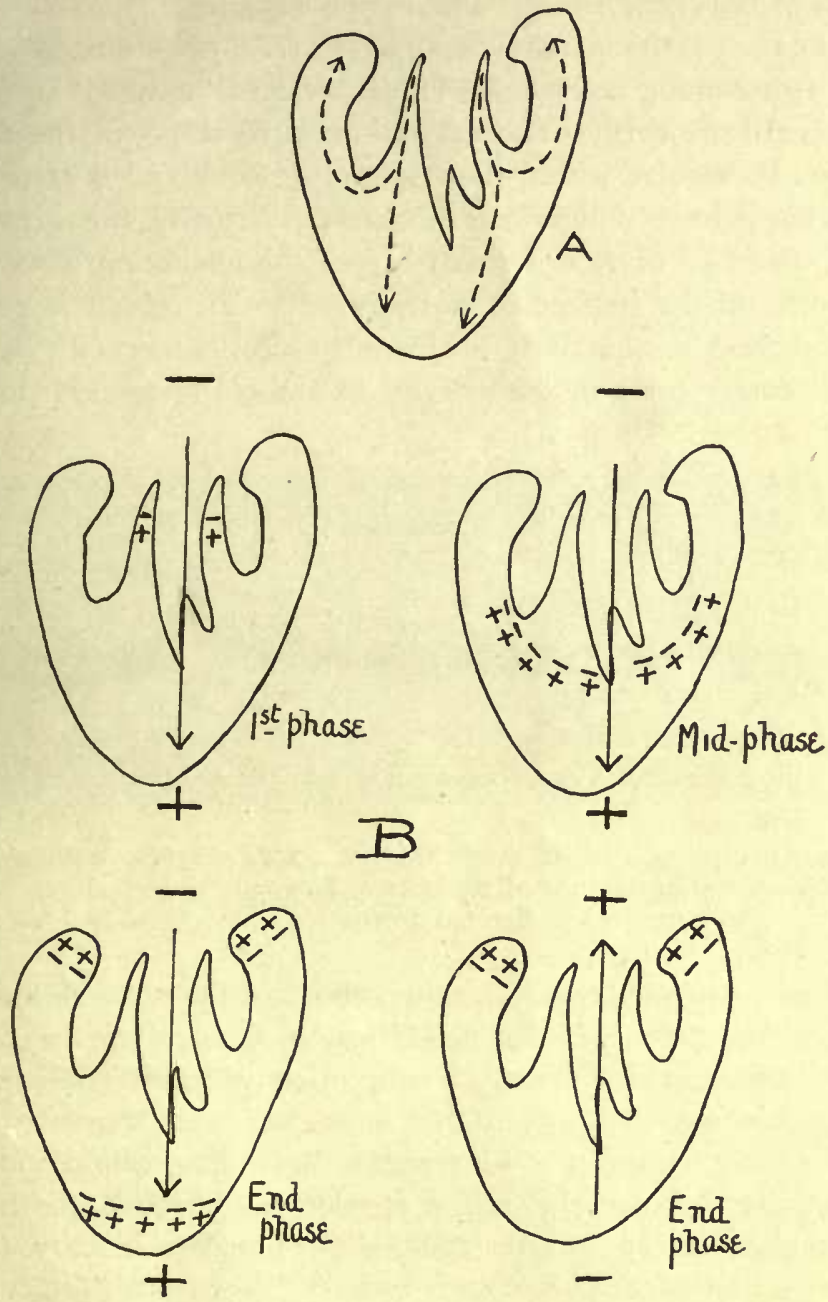

Fig. 6.-Course of excitation wave in activation of ventricle.

negativity at the apical contact necessarily means activity of the muscle lying nearer to the base than the apex of the ventricle. The direction of this deflection is what it is, because the excitation wave begins in the lower part of the bulbus (i. e., toward the apical contact) and the inactive muscle in its vicinity lies above it (i. e., toward the basal contact). 
The position of the bulbus, relative to the rest of the ventricular muscle with which it is in functional union ${ }^{\tau}$ does not affect the direction of the deflection.

Consider now the initial deflection $(R)$ or deflections ( $R$ and $S$ ). The meaning of $R$ according to my hypothesis is not obscure. It is due to set of the current in the axis of the heart, $i$. $c$. from above downward, over the greater part of that phase of the cycle during which the ventricle is becoming activated. The ventricle is activated in the gross from above downward. The excitation wave starts in the muscular funnel and trabeculae within the heart (Fig. 6A) and is at first propagated wholly in a downward direction. Actually the greater part or entire upstroke of $R$ is written before the excitation wave appears at any point on the surface of the ventricle. To regard $R$ as an evidence of general basal activity is obviously inconsistent with this observation; $R$ corresponds to the activity of muscle lying deep inside the

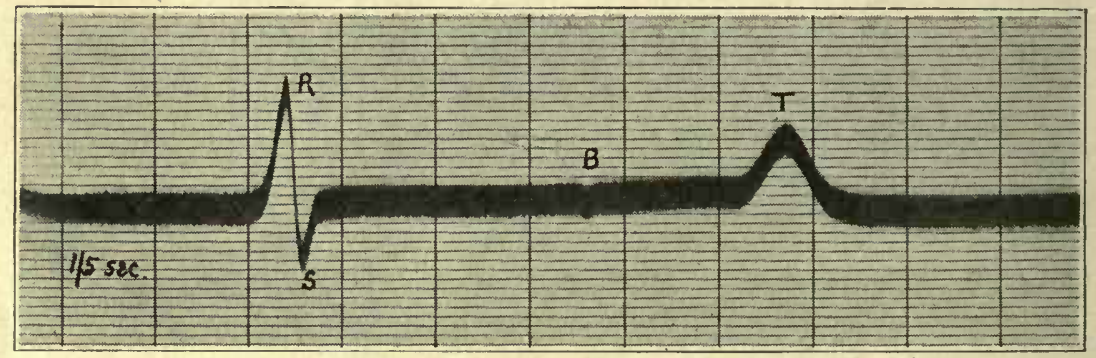

Fig. 7.-An electrocardiogram taken from a toad's heart; leading from the mouth and abdomen in the axis of the heart. The deflection produced by activity of the bulbus arteriosus $(B)$ is directed downward.

central portions of the heart. The hypothesis of limited potential differences applied to the amphibian ventricles provides us with a clear conception of the electrical events, a conception which is consistent with the manner in which the excitation wave has been shown to spread. During the early phases of $R$ 's inscription the muscle is becoming active in the central trabeculae, this region is relatively negative, the relatively positive region lies apexwards and in its immedate vicinity (Fig 6B 1st phase). As the excitation wave proceeds downward, the advancing border spreads laterally, but the balance of the potential differences manifests itself still in the same direction (Fig. $6 \mathrm{~B}$ midphase). In its

7. The reply which has been made, that there is a natural line of block between the two chambers, does not appear to me relevant. They are united functionally by muscle fibers, the region of block being simply a region of slow propagation; if it is held that this region of slow propagation constitutes a line of complete separation from the standpoint under consideration, the burden of proving this lies with those who hold the view. 
further progress the advancing border of the wave is not only proceeding toward the apex, but is now also traveling back in the lateral walls to reach the base (Fig. $6 \mathrm{~B}$ end-phase left-hand figure). Activity in the region of the apex still tends to maintain the electric axis from base to apex; the basal activity tends to set it in a contrary direction. But the effect of this basal activity is apparently insufficient to upset the average direction, which still remains from base to apex. The basal contact is throughout relatively negative to the apical contact. If, however, as often happens in the amphibian heart, the basal segments of muscle are the last supplied, this opposition of the apical effects is removed and the effect of basal activity then appears It manifests itself in the form of an $S$ wave, a downward deflection indicating relative negativity of the apical contact. The axis of the electromotive force is now from below upward (Fig. $6 \mathrm{~B}$ end phase, right-hand figure).

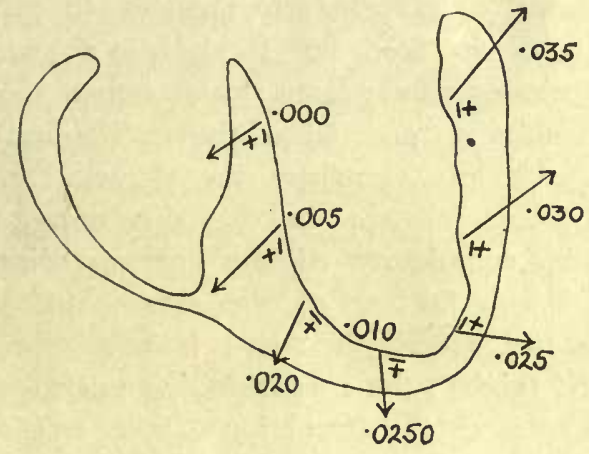

Fig. 8. - Times of arrival of excitation wave relative to each other indicated in decimal points of a second.

Thus, our hypothesis brings us to a reasonable explanation of a curious but actual observation, namely, that the appearance of an $S$ wave in the electrocardiogram of amphibians is associated with late arrival of the excitation wave at the ventricular base. No other present day hypothesis will afford an explanation of this phenomenon. This example is in my view precisely comparable to that of the bulbus already cited. In both instances basal activities are responsible for relative negativity of the apical contact; but the present illustration has an advantage over the first, in that there can be no question here of separate muscle systems; the whole of the musculature of the ventricle is one uniform syncytium.

\section{THE MAMMALIAN ELECTROCARDIOGRAM}

Similar methods of observation are applied to the mammalian ventricle and, although in this instance we deal with a far more complex arrangement, similar correlations can be shown to exist between the manner in which the excitation wave spreads and the set of the elec- 
trical axis from instant to instant. To consider the constitution of this electrocardiogram in full detail would detain us too long, it must suffice if by a single example I illustrate again the general principle of interpretation, and show that the hypothesis of limited potential differences is applicable in this case also. Let us take the simpler case, the excitation of the dog's left ventricle.

The excitation wave is found by observation to start in the septum of the ventricles; it is traced down the septum to the apex and from the latter up the lateral wall to the base. The times of arrival, relative to each other, are indicated in Figure 8 in decimal points of a second. But in moving along this semicircular path the wave does not proceed in a direction parallel to the borders of the muscular track, it is penetrating the wall in successive segments of its course; it moves always from within outward along centrifugal paths; each part of the wave travels from the endocardial to the pericardial surface. Throughout its passage, the electrical axis constantly changes so that, while in the initial stages this axis sits from left to right in the body, it gradually shifts to a base-apex direction; from this it swings more and more to the left until, eventually, and when the excitation wave is reaching the base of the left ventricle, the electrical axis is directed chiefly upward. This change in the direction of the axis is responsible for the complexity of the corresponding electrocardiographic curves. When the set of the axis is in the main downward, a basal contact (right shoulder) is relatively negative to an apical contact (left thigh); the corresponding upstroke in the electrocardiogram contributes to the formation of the well-known deflection $R$. On the contrary, when the set of the axis is mainly, upward, the apical contact becomes relatively negative to the basal contact and the corresponding downstroke in the electrocardiogram is the chief constituent of the well known deflection $S$. As in the amphibian heart, $S$ does not represent an apical activity, it represents a basal activity. Now this example, though complex, is a striking one. There is, within the reasonable bounds of error in observation, complete correspondence between the readings which indicate the distribution of the advancing excitation wave and the set of the electrical axis at corresponding phases of the cycle, when the set of this axis is read in terms of my hypothesis of limited potential differences. These observations are in full accord with our knowledge of the anatomy of the ventricle, in accord with a distribution of the excitation wave through the left division of the auriculoventricular bundle and its arborization; for the left division enters the ventricle on its septal surface and distributes its branches to the septum, to the apical parts of the heart and to the lateral walls of the heart and to the lateral walls at the base, in this order. Consider the ascertained order of spread and arrange theoreti- 
cally the electrical axis, using the hypothesis of distributed potential differences, and one arrives at a result which is not consistent with observation. According to this hypothesis, relative negativity should appear, first of all, at the basal contact; secondly when the wave of excitation reaches the apex, the apical contact should appear relatively negative; finally when the wave returns to the base, the basal contact should again show relative negativity. The second and last suppositions are not in accord with the facts. But if, on the other hand, one arranges the potentials theoretically, using the hypothesis of limited potential differences, harmony exists.

In the light of these observations and in the light of those previously described, I have no hesitation in discarding the older hypothesis and in accepting in its place the hypothesis which I term that of limited potential differences. It is in accord, so far as can be seen, with all present observations on heart muscle. I do not claim that it affords a final explanation, being quite prepared to find that future observations will necessitate its modification; but it is desirable, nay it is essential, that we should possess ourselves of an hypothesis which harmonizes with all present day observation; an hypothesis which will serve to direct further experiment along fruitful channels. Hypotheses which are out of harmony with present observations will not serve this purpose. The present hypothesis has been put forward and is now emphasized in the belief that it will supply our present and immediate needs. 





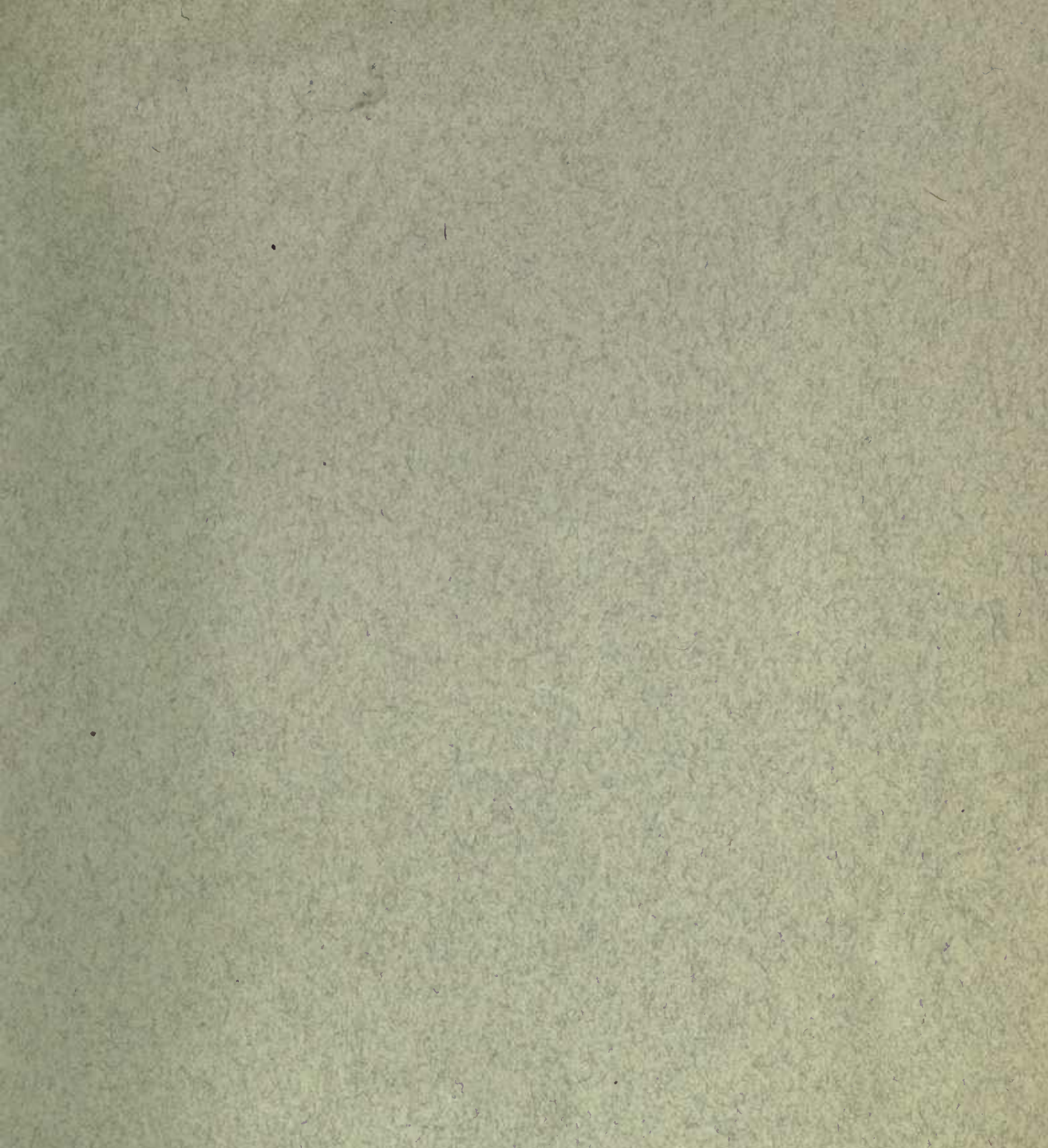


186 +4a
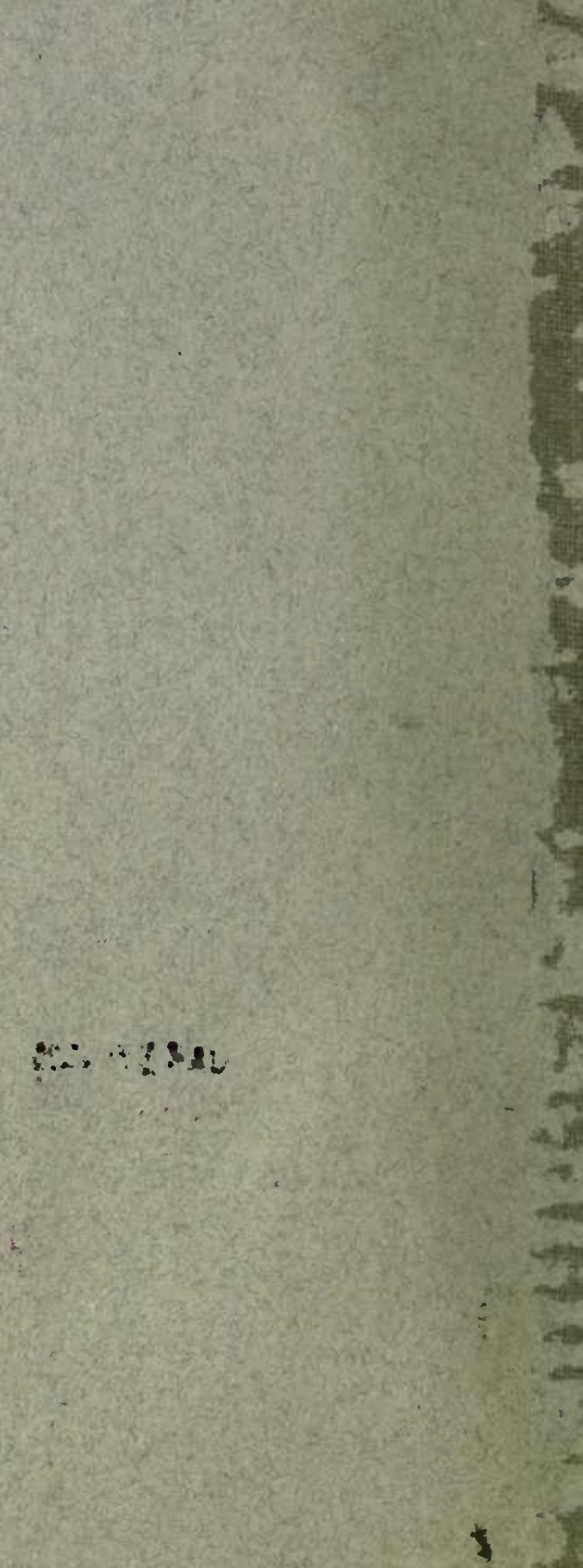

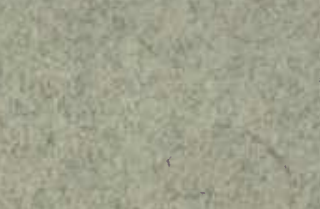

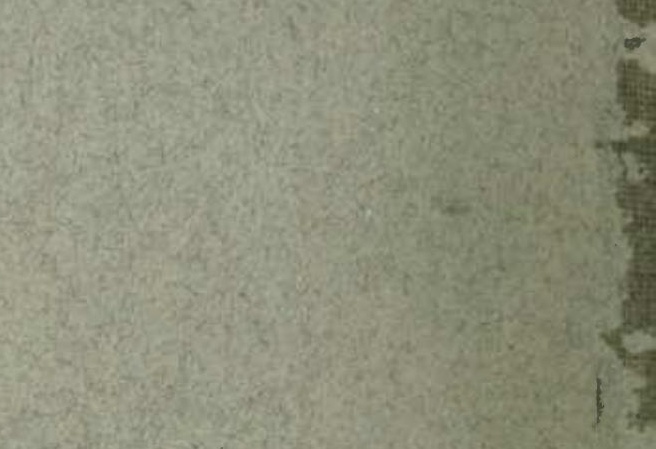




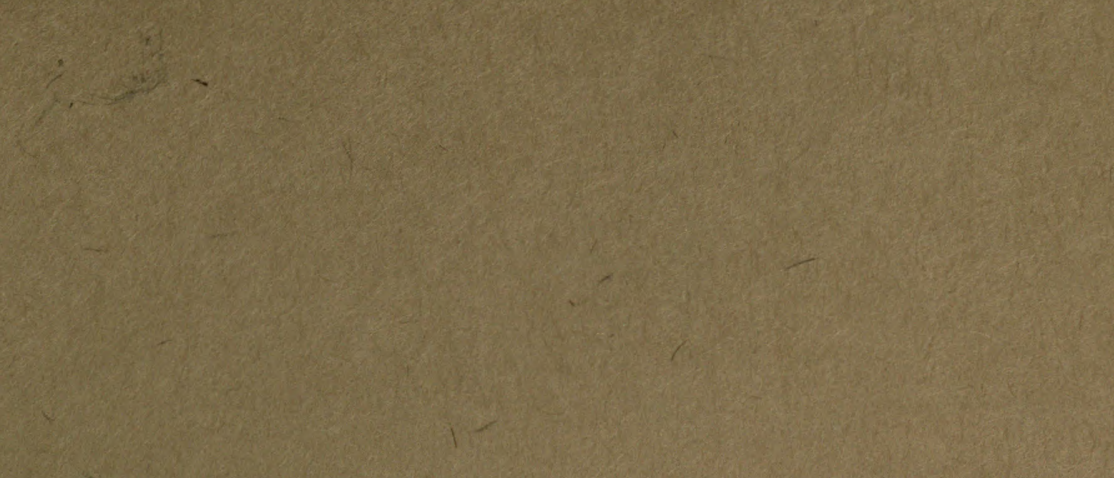

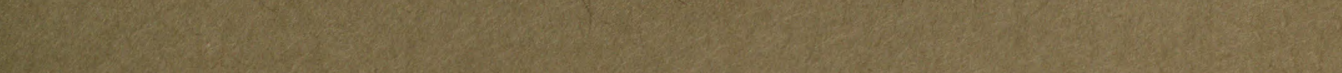

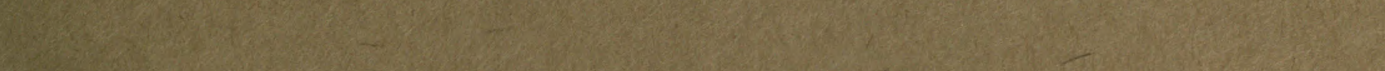

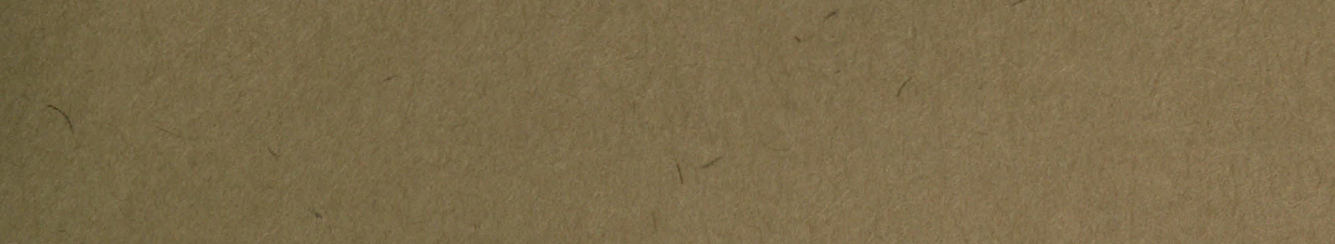

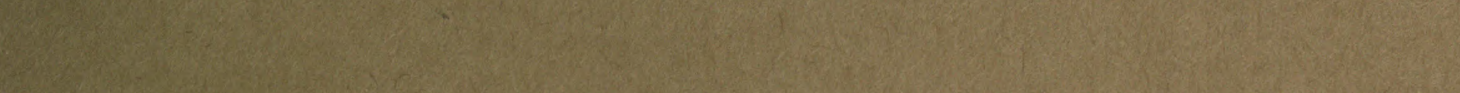
(3)

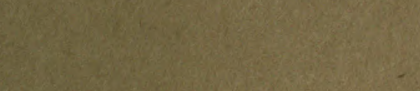

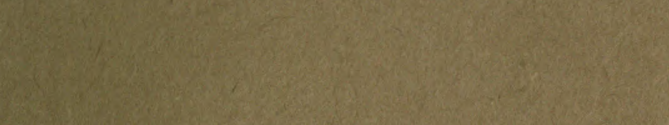
W.

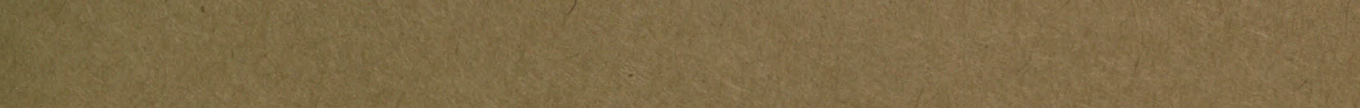

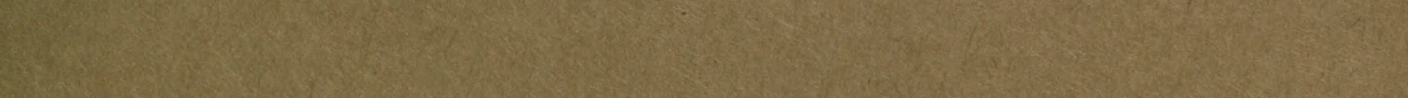

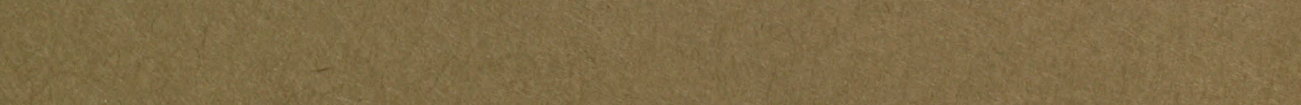

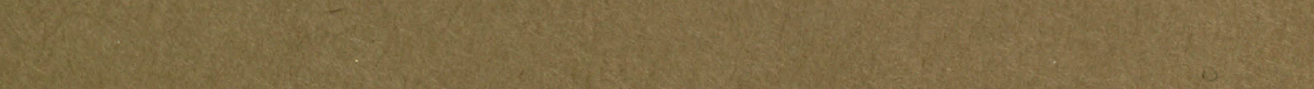

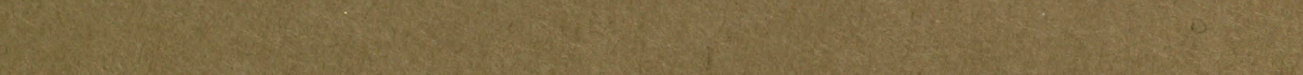
2.

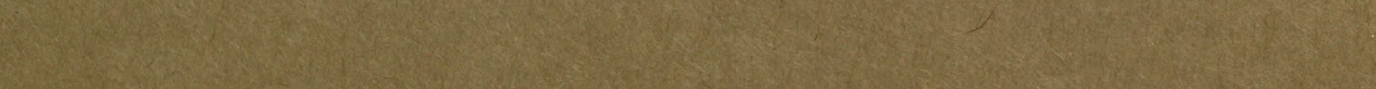

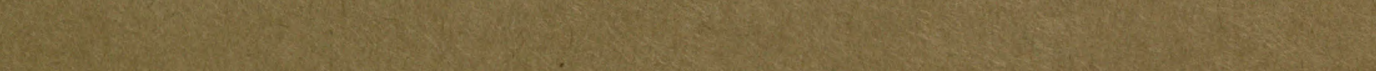

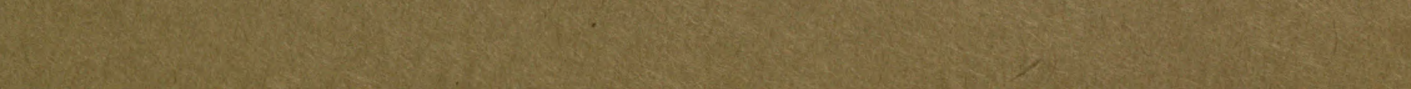




